\title{
B. Mutlu Sumer: Liquefaction around marine structures
}

\author{
World Scientific, Advanced Series on Ocean Engineering-Volume 39
}

\author{
Shinji Sassa
}

Received: 24 July 2014 / Accepted: 17 September 2014 / Published online: 11 October 2014

(C) Springer International Publishing AG 2014

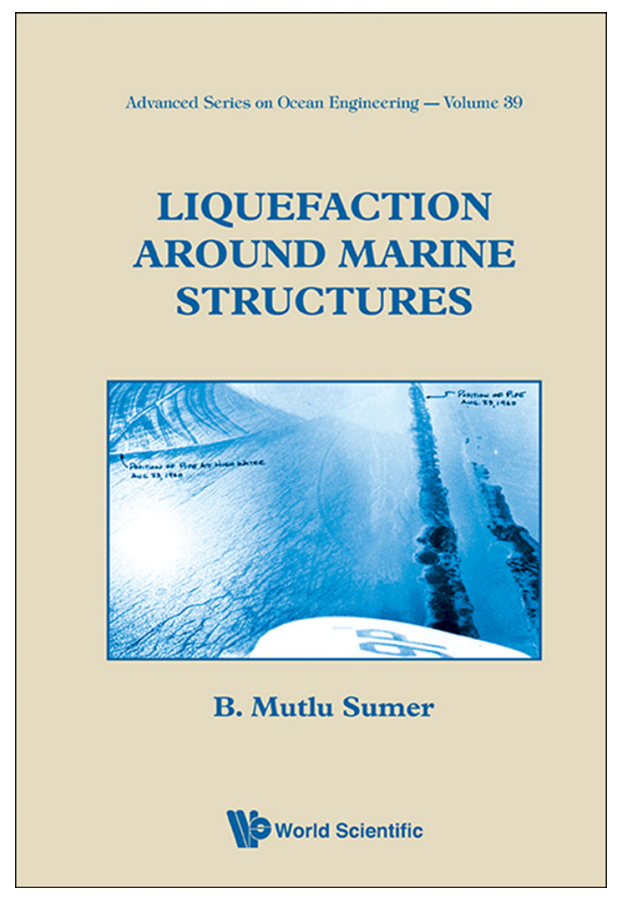

Liquefaction is a phenomenon that turns soil as solid into a semifluid state as induced by earthquake, wave loading or other environmental forcings. Once liquefaction occurs, it has considerable consequences on the stability of the surrounding structures, since it diminishes the bearing capacity of the soil. While plenty of books are available on the earthquake-induced liquefaction, there has been no concrete

Communicated by Umesh A. Korde.

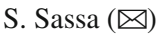

Head of Soil Dynamics Group and Research Director of Asia-Pacific Center for Coastal Disaster Research, Port and Airport Research Institute, 3-1-1 Nagase, Yokosuka 239-0826, Japan e-mail: sassa@ipc.pari.go.jp book addressing the problem of ocean wave-induced liquefaction.

This is a well-written and comprehensive book describing the physics and processes of seabed liquefaction around marine structures. The primary focus of the book is placed on wave-induced liquefaction; however, a concise yet sufficient coverage is given to describing the implication of earthquakeinduced liquefaction for marine structures. The state-of-theart knowledge on seabed liquefaction has been decently collected based on the author's 20 years of research and the work of other prominent researchers from both geotechnical and hydrodynamic perspectives.

The book consists of 11 chapters in 453 pages. The first chapter briefly introduces and explains the physics of liquefaction under ocean waves and earthquakes. It also gives a brief account of several case histories of seabed liquefaction and its consequences, which surely attract the interest of the readers. The basic Biot consolidation equations and their solutions with classical poro-elasticity theory are introduced in Chap. 2, so as to describe wave-induced shear stresses and pore water pressures in a seabed soil under progressive and standing waves. Two major mechanisms of wave-induced liquefaction, namely, residual and momentary liquefaction are described in detail in Chaps. 3 and 4, respectively. Here, residual liquefaction accompanies the build-up of pore water pressures with an analogy with earthquake-induced liquefaction. By contrast, momentary liquefaction occurs periodically under wave trough in the case of unsaturated soils. In these chapters, the author succeeds in conveying the richness of the physics involved in the sequences of soil behavior during wave-induced liquefaction and their state-of-the-art predictions. Subsequent chapters are devoted mainly to describing the processes, impacts and modelling of the seabed liquefaction around marine structures, such as floatation and sinking of pipelines in Chaps. 5, 6, liquefaction at gravity struc- 
tures in Chap. 8, and the stability of rock berms in liquefied soil in Chap. 9. The susceptibility of liquefaction under standing wave in comparison to that under progressive wave is highlighted in Chap. 7. Earthquake-induced liquefaction with focus on Japanese and Turkey experiences and existing models of the liquefaction-induced ground deformations as well as the impact of tsunami on coastal structures are described in Chap. 10, together with the reviews of the existing design codes and guidelines. Finally, a range of countermeasures against seabed liquefaction are summarized in Chap. 11.

Standard wave flume tests have been used to obtain insights into wave-induced seabed liquefaction. However, there remains a problem such that time-scaling laws for such $1 \mathrm{~g}$ wave-soil testing have not yet been established. A notable distinction of this book is that the author discusses and validates the relevant results in light of geotechnical centrifuge wave-soil modelling that has been shown to match the timescaling laws for fluid wave propagation and consolidation of the soil. This fundamental yet important issue further supports the clarity dealt within this book.
A total of 18 design examples and numerical exercises are presented throughout the chapters, as complemented by a description of some standard methods to assess in situ liquefaction potential in an Appendix. These certainly make it easier for the readers, as the the author states, "to assess liquefaction potential, to make engineering predictions, and to make recommendations as to how to mitigate potential risks". The author also sensibly avoids a discussion of advanced elastoplastic constitutive modelling of soil and illustrates several useful and workable mathematical approaches for the sake of coastal and ocean engineers.

A CD-ROM containing five good videos is attached to the book. These materials would also greatly assist the readers to understand the phenomena of wave-induced liquefaction.

Overall, this book is highly recommended for all professionals and researchers interested in seabed soil liquefaction and the stability of marine structures, and is indeed suitable as a textbook for graduate/postgraduate students in this field. 